\title{
Learning Assistant Identity Development: Is One Semester Enough?
}

\author{
Jessica Conn, Eleanor W. Close, and Hunter G. Close \\ Department of Physics, Texas State University, San Marcos, TX 78666
}

\begin{abstract}
The physics department at Texas State University has completed five semesters with a Learning Assistant (LA) program and reform-based instructional changes in our introductory course sequences. We are interested in how participation in the LA program influences LAs' identity both as physics students and as physics teachers; we have previously reported trends in increased community involvement and a shift in experienced LAs' concepts of what it means to be competent. Our interview data now include first-semester LAs, and we see a significant difference in physics identity development between these LAs and those with more experience. LAs near the end of their first semester seem to be experiencing a state of unease with respect to teaching and learning. We explain this discomfort in terms of Piagetian disequilibrium: their conceptions of competence in teaching and learning have been challenged, and they have not yet constructed a new model.
\end{abstract}

Keywords: Learning assistants, physics identity, community of practice, teacher preparation, teacher recruitment.

PACS: 01.40.Fk, 01.40.Ha, 01.40.jc

\section{INTRODUCTION}

The Learning Assistant (LA) model, first developed at the University of Colorado-Boulder in 2003 , has been documented to lead to a number of positive effects on both LAs and students in LAserved courses [1]. The physics department at Texas State University has recently implemented an LA program supporting course reform in our introductory calculus-based physics sequence. Our LA program has expanded from a one-section pilot with six LAs in spring 2012 to serving all sections of the three-course calculus-based introductory physics sequence with 25 LAs in spring 2014. LAs are recruited from undergraduate STEM majors, attend weekly preparation sessions, and assist instructors with interactive instructional activities (primarily tutorials [2]) during regular class time. In addition, new LAs take a course in physics cognition and pedagogy, and some LAs work in the Physics Help Center (a drop-in tutoring center) and grade papers.

In previous work we have analyzed the LA experience empirically in terms of the existing theoretical frameworks of communities of practice and physics identity $[3,4]$. Specifically, we have examined written and video data sources for evidence of whether participation in the LA program has affected elements of LAs' identity, and if so, in what ways and through what program elements. In this paper we compare interview data from experienced and novice LAs to examine the timescale of observed changes in LA identity, with a focus on differences in self-perceptions of competence.

\section{THEORETICAL FRAMEWORK}

We briefly describe the two theories named above, and build a correspondence between factors in the physics identity framework and community of practice theory. We then use this blended theory to analyze video of LA interviews and written artifacts.

\section{Identity in Practice}

Lave and Wenger describe development of identity and participation in communities of practice as deeply intertwined [5, 6]. Members of a well-functioning community of practice experience "mutual engagement, a joint enterprise, and a shared repertoire" [6]. Participation in a community of practice shapes and is shaped by the ways in which members of the community engage each other around their shared practice. Identity, as shaped by this engagement in practice, is not an inherent or fixed quality of a person but rather a process of continuous re-negotiation. Within this framework, Wenger [6] identifies five characterizations of identity. In this paper we will focus on one of these: identity as community membership. 


\section{Physics Identity Self-Concept}

Hazari and colleagues describe and empirically validate a theoretical framework for physics identity composed of three dimensions: personal interest, student competence / performance, and recognition by others $[7,8]$. Assessment of physics identity is based on self-report, e.g., the dimension competence can be described as "belief in ability to perform required physics tasks," and is measured through survey items such as "I understand concepts I have studied in this subject" and "Others ask me for help in this subject" [8]. Regression models from these studies show that the physics identity construct strongly predicts intended choice of a career in physics.

\section{Relating Self-Concept and Practice}

In order to relate the empirically tested physics identity framework to concepts from the more general community of practice theory, we build a correspondence between factors. Community membership defines identity through the forms of competence developed and valued by participants in the community, including ways of interacting and working together, shared interpretations and perspectives, and ability to make use of a shared repertoire of language, tools, and other resources. A self-reflection on identity characterized in this way might be "Do I know how to do things this community values?" This encompasses the physics identity dimension of competence / performance. This aspect of the blended identity theory is the focus of analysis in this paper; other aspects are described in previous work $[3,4]$.

\section{METHODS}

We examine written data sources and video of LA interviews for evidence of ways in which participation in the LA program has affected elements of LAs' identity. Written data are drawn from multiple sources, including teaching reflections, program applications, and written programmatic feedback. A subset of experienced LAs (approximately 30\%) have participated in loosely-structured clinical interviews; in addition, we have conducted interviews with three novice LAs near the end of their first semester in the program. Interview subjects were selected to include diversity of major, gender, career plans, and length of experience in the LA program. The interview protocol included questions probing both self-perceptions (e.g., What parts of being an LA are you particularly good at? Has being an LA made you more competent at other things besides teaching?) and practice (e.g., Do you use the Help Center to study or hang out? Do you interact differently with faculty since becoming an $L A ?$ ).

Video data from interviews was collaboratively analyzed for insight into the impact of the LA program on participants' construction and perception of identity. Adjustments were made to the interview protocol both during individual interviews, in response to the participant's experiences and reflections; and between interviews, in response to emergent themes in the data.

\section{ANALYSIS}

In both written and video data, experienced LAs express a strong sense of increased competence through their participation in the program, both generally ("Being an LA has made me a more competent person all around") and in specific contexts such as solving physics problems and helping students. This was not initially surprising to us: we expect the experience of re-visiting introductory physics concepts in a highly interactive and supported environment to increase LAs' skills with the material and their feelings of competence in working with it. The more surprising aspect of the shift described by experienced LAs in their interviews is a change in what they identify as demonstrations of competence. We describe this re-defined concept of competence below, and compare it to the reflections of novice LAs.

\section{Experienced LAs and Competence}

Through their participation in the community of physics instructors, LAs expand their repertoire both of what they know how to do and of what they see as valuable in physics. Most experienced LAs comment during their interviews on how this has impacted them beyond the immediate functions of their role as an LA. For example, Mike (pseudonym), a computer science major and third-semester LA, describes how being an LA has made him a better learner (" $\mathrm{J}$ " is the interviewer and first author):

J: So, you said that the LA program made you a better communicator - did it make you better at other things, too?

M: Um...I mean, it made me better at physics, that's for sure. Um... I don't...It's - It's made me a better learner, as well.

$\mathrm{J}$ : Tell me about that.

M: So - part - I mean, to be a tutor, you have to be able to put things into - into definitions that you wouldn't necessarily use. [...] so it teaches you to have to be a bit more vicarious and think about the way other people see things. And in general $[\ldots]$ 
what I think that created for me was metacognition - the ability to think about how $I$ think about things, or how people in general think about things. And that, to me - If I'm struggling with a concept, I can think about, you know, what am I doing wrong here, why am I not thinking about this correctly, what can I do differently to understand this concept. ... and I think that's directly related to teaching in the LA program. [...] I think the ability the LA program has given me [is] when I'm studying on my own, to treat myself as both the tutor and the tutee. From the tutor perspective, look at myself as a tutee and say, okay, what am I struggling to understand here. Can I ask myself questions that I would ask a tutee, and derive [...] where the failure to understand actually lies.

Mike describes how being an LA has given him tools to manage his own learning, particularly in times when he doesn't yet understand a concept. In other interviews, experienced LAs explicitly discuss learning that it's okay to be wrong. In the interview excerpt below, Alan, a junior-level physics major in his third semester as an LA, describes this:

J: What do you like most about being an LA? Why do you continue to - yeah, what do you like most about it?

A: Um... I just feel like it's changed the way that I try to learn things. [...]

J: Right

A: So...and I think it's helped me be okay with being wrong, as well. Which is a lot less frustrating when I'm doing homework - well, I'm still frustrated when I do homework, but... when I'm helping people in Help Center, and I'm not sure what the answer is, I'm okay with getting another LA and working through it with the student...

\section{J: Right}

A: ....and I think that makes them more comfortable asking questions, because they don't - they're not intimidated by us as much as they are when the instructors are there... because they feel like we're on the same level as them.

J: Right - like if it's okay for us to be wrong, then it's also okay for them to be wrong,

A: Mmm-hmmm

J: ...so it's not so scary.

A: Right.

This discussion suggests that his participation in the practices of the LA community has caused Alan to shift away from a more correct-answer-oriented, "school-physics" definition of competence to one that is inclusive of competent and valuable ways of interacting with other members of the community, including interactions around incorrect physics ideas.
Another LA, Gavin, also identified being wrong not only as okay but as potentially valuable. The context of the question was the weekly content preparation session, during which LAs and faculty work through curricular materials. The interviewer asked Gavin: "Did you ever see professors make a mistake? Was it normal, or weird?" Gavin responded:

"We were just used to it by then - I feel like one of the goals of the LA program is to... to teach students that it's okay to make mistakes, and it's even good, if you can recognize that you're making mistakes. So - it was no big deal."

Both Gavin's and Alan's responses suggest a shift away from a model of competence centered on obtaining correct answers. Instead they have learned to value and enjoy interactive, logical exploration and argumentation. This practice re-purposes being wrong (or saying wrong things) from a form of incompetence to an important component of competent engagement, within a practice that recognizes multiple forms of competence.

\section{Novice LAs and Disequilibrium}

We have conducted interviews of three novice LAs near the completion of their first semester of participation in the LA program. These LAs vary in academic standing from sophomore to senior. Like the more experienced LAs, they described improving their understanding of physics and their skill in guiding introductory students in learning situations. However, in response to the same interview protocol, none of the novice LAs made the same kinds of connection described above between the LA experience and their own skill as a learner, and none of them described the same transformation in their sense of what it means to be competent and how being wrong can be a form of competent participation.

For example: Hannah, a sophomore engineering major, responds to a question about improving her skill at working with students in the Help Center by describing the value of struggle in the problem-solving process:

J: What do you think has changed inside of you to make it... make you handle those situations better?...

H: Um, I would say, part of it was confidence, and part of it is learning more physics, and then another part was, um... Dr. Close one day in the pedagogy class, she told us that we're not there to be solution manuals. And that, you know, we don't have to know all the right answers, and we're not there to help them just get the right answer, we're there to help them think about the problems. And so, once I started really 
believing that and listening to that, and reminding myself while I was helping people, that changed my outlook a lot $[. .$.$] because, you$ know, it's okay to make mistakes, and it's okay to mess up, as long as you still try to help them.

J: Mmm-hmm. That's awesome. Umm... Why do you think that... just... helping them is good enough? As opposed to being solutions manual. I mean... that $i$ s enough, but where do you think that comes from? Why do you think that's okay?

$\mathrm{H}$ : I'd say because of my struggles as a student in some difficult subjects. Like, even if a tutor couldn't help me get to the right answer, if they at least helped me in the thought process about it, then, $[\ldots]$ maybe eventually I would find the right answer, or we would work towards it together. And through the struggle of us both being unsure, we were able to, um, find the answer in the end, and then I remember it more, because there was a longer struggle through it.

Here we see that Hannah talked about value of questions, and described it as okay to not know the answer, but in the limited context of the tutoring situation: the desired competent performance seems to be in remembering the answer when you eventually get there. We find no evidence of belief that discussion or argumentation is a form of competence in and of itself. Note that unlike Mike, Hannah does not reflect on changes in her own learning process, except to the extent of encoding learning into her memory.

Hannah's continued emphasis on correct responses is reiterated in another exchange near the end of the interview:

J: Do you think you learned skills here at... in the LA program that will carry over into helping you...get what you need [as a student]?

$\mathrm{H}$ : Mmm-hmm, definitely. Umm, I think... last semester, being the one who was tutored all the time, and then this semester becoming a tutor, I was able to see it from both sides. And, um, now... I 'd be able to better pick [...] the type of help that I wanted. Because... in math, if I would go get tutored one-on-one outside of class, that would help a lot for me to get through that homework, but I wouldn't always remember it. [...] But in Physics 1, [...] having the group discussions really helped a lot because if there was someone in my group who was arguing with me, like, saying that I'm wrong and they're right, and then if I keep thinking I'm right, and we talk about it more, and I really am wrong, then I'll remember that.

J: Right.

$\mathrm{H}$ : And if I really am right, I'll remember it too.

The other novice LAs either described a similar relationship to correct answers, or restricted their responses to teaching-related skills and did not demonstrate integration of their experiences as an LA into their conceptions of competence as a learner.

\section{IMPLICATIONS}

Extended participation in the LA program seems to support an expansion of LAs' perception of what constitutes competence: they learn to value and enjoy multiple ways of engaging in the physics community, and specifically to re-purpose saying wrong things from a form of incompetence to an important component of competent engagement. This expanded definition of competence increases opportunities to identify as a competent member of the community, which strengthens physics identity.

Given the positive relationships between perceptions of competence, physics identity, and intentions to persist in physics [7,8], our data suggest a possible mechanism for the transformative nature of the LA experience [3]. The novice LAs we interviewed, however, did not demonstrate this shifted perception of competence; the data suggest that this transformation takes up to three semesters to occur. Additional interviews with LAs as they progress from novice to experienced are needed to confirm this comparison. A question for further research is whether an explicit focus during the LA experience on models of competent engagement could prompt this shift to occur earlier.

\section{ACKNOWLEDGMENTS}

This work and the Texas State University Physics LA Program are supported in part by NSF grant DUE1240036 and the Halliburton Foundation.

\section{REFERENCES}

1. V. Otero, S. Pollock, \& N. Finkelstein, Am. J. Phys. 78 (11), 1218-1224 (2010).

2. L.C. McDermott, P. S. Shaffer, \& U.W. PEG, Tutorials in Introductory Physics, $1^{\text {st }}$ ed., Pearson, 2002.

3. E. W. Close, J. Conn, \& H. G. Close, in AIP Conf. Proceedings: PERC 2013, 2014, pp. 89-92.

4. E. W. Close, J. Conn, \& H. G. Close, in Learning and becoming in practice: ICLS 2014, pp. 1533-1534.

5. J. Lave \& E. Wenger, Situated learning: Legitimate peripheral participation, Cambridge Univ. Press, 1991.

6. E. Wenger, Communities of Practice: Learning, Meaning, and Identity, Cambridge Univ. Press, 1998.

7. Z. Hazari et al., J. Res. Sci. Teach. 47 (8), 978-1003 (2010).

8. R. M. Lock, Z. Hazari, \& G. Potvin, in AIP Conf. Proceedings 1513 (PERC 2012), 2013, pp. 262-265. 